\section{SOI: $1.1 /$ TAS DOI: $10.15863 /$ TAS}

International Scientific Journal Theoretical \& Applied Science

p-ISSN: 2308-4944 (print)

e-ISSN: 2409-0085 (online)

Year: $2016 \quad$ Issue: 1 Volume: 33

Published: $30.01 .2016 \quad \underline{\text { http://T-Science.org }}$

SECTION 7. Mechanics and machine construction.
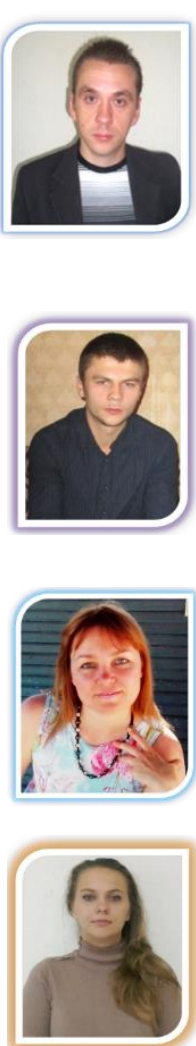

Denis Alexandrovich Chemezov Master of Engineering and Technology, Corresponding member of International Academy of Theoretical and Applied Sciences, Lecturer of Vladimir Industrial College, Russian Federation chemezov-da@yandex.ru

Vasily Sergeevich Seliverstov Student of

Vladimir Industrial College, Russian Federation seliverstov-vasily2015@yandex.ru

Anzhelika Vladimirovna Bayakina Lecturer of

Vladimir Industrial College, Russian Federation

bajakina.anzhelika@yandex.ru

Natalia Andreevna Zezina Student of

Vladimir Industrial College, Russian Federation natalia162743@gmail.com

\title{
THE INFLUENCE OF THE MAGNITUDE OF THE RADIUS CHAMFER IN THE DIE HOLE ON THE DEGREE OF DEFORMATION OF THE PROCESSED MATERIAL AND THE PRODUCTIVITY OF THE DEEP DRAWING PROCESS OF THE PLATE STOCK
}

Abstract: The article gives recommendations on optimization of deep drawing of the plate stock based on the obtained dependencies productivity treatment and the degree of deformation of the material from the magnitude of the radius chamfer in the die hole.

Key words: a deep drawing, a plate stock, a radius chamfer, a deformation, a die, a productivity.

Language: Russian

Citation: Chemezov DA, Seliverstov VS, Bayakina AV, Zezina NA (2016) THE INFLUENCE OF THE MAGNITUDE OF THE RADIUS CHAMFER IN THE DIE HOLE ON THE DEGREE OF DEFORMATION OF THE PROCESSED MATERIAL AND THE PRODUCTIVITY OF THE DEEP DRAWING PROCESS OF THE PLATE STOCK. ISJ Theoretical \& Applied Science, 01 (33): 52-57.

Soi: http://s-o-i.org/1.1/TAS-01-33-11 Doi: crossef http://dx.doi.org/10.15863/TAS.2016.01.33.11

УДК 621.7.043

ВЛИЯНИЕ ВЕЛИЧИНЫ РАДИУСНОЙ ФАСКИ В ОТВЕРСТИИ МАТРИЦЫ НА СТЕПЕНЬ ДЕФОРМАЦИИ ОБРАБАТЫВАЕМОГО МАТЕРИАЛА И ПРОИЗВОДИТЕЛЬНОСТЬ ПРОЦЕССА ГЛУБОКОЙ ВЫТЯЖКИ ЛИСТОВОЙ ЗАГОТОВКИ

Аннотация: В статье даны рекомендации по оптимизации процесса глубокой вытяжки листовой заготовки на основании полученных зависимостей производттельности обработки и степени деформации материала от величины радиусной фаски в отверстии вытяжной матрицы.

Ключевые слова: глубокая вытяжка, листовая заготовка, радиусная фаска, деформация, матрица, производительность.

При невысокой точности размеров и геометрической формы детали обработка давлением пластических материалов занимает лидирующее место в мало- и безотходном 
производствах. Для получения тонкостенных полых деталей типа «стакан», «крышка», «корпус» и др. применяется листовая штамповка и, в частности, формообразующая операция вытяжка. Образование контура детали происходит при вдавливании листовой заготовки в рабочие поверхности формообразующих инструментов (пуансон и вытяжная матрица). За счет пластической деформации обрабатываемый материал принимает форму этих поверхностей.

В работах $[1,2]$ определено, что при толщине листовой заготовки более 2.5 мм процесс гофрообразования не происходит, т. е. вытяжку материала можно производить без дополнительных прижимных устройств. Заготовки толщиной до 2 мм [3] обладают малой жесткостью, и для выполнения процесса глубокой вытяжки детали необходимо применять складкодержатель.

Рассмотрим следующие вопросы, касающиеся оптимизации технологического процесса глубокой вытяжки полых деталей, которые имеют форму тел вращения.

Первый вопрос. Влияние степени напряженно-деформированного состояния обрабатываемого давлением материала на качество готовой детали.

Наибольшая деформация материала листовой заготовки происходит в зоне перехода плоской поверхности в рабочее цилиндрическое отверстие вытяжной матрицы [4, 5]. Дальнейшее продавливание заготовки пуансоном приводит к утончению боковых стенок и дна детали [6] и образованию в местах изгибов трещин, которые могут привести к частичному разрушению материала. Исследование интенсивности деформации материала в условиях изменения величины радиусных фасок в отверстии матрицы позволит определить оптимальный размер конструктивного элемента формообразующего инструмента, при котором в готовой детали возникают минимальные остаточные напряжения [7].

Второй вопрос. Повышение производительности технологического процесса вытяжки.

Повышение

производительности достигается путем увеличения скорости пуансона. При этом увеличивается скорость деформации материала заготовки, а, значит, и сопротивление деформации [8]. Это требует повышения усилия, которое действует на пуансон штампа. Можно сократить основное время на обработку детали путем подбора рациональной геометрии формообразующих инструментов без изменения технологических режимов пластического деформирования заготовки.

Исследования влияния величины радиусной фаски в отверстии матрицы на степень деформации обрабатываемого материала и производительность процесса глубокой вытяжки листовой заготовки осуществлялись в многоцелевой компьютерной программе конечноэлементного анализа LS-DYNA.

Были выполнены 20 экспериментов, в которых режимы обработки не изменялись на всем временном диапазоне процесса глубокой вытяжки полой детали. Имитационное моделирование динамики процесса глубокой вытяжки производилось в трехмерной постановке.

Процессу вытяжки в холодном состоянии подвергали листовые заготовки, изготовленные из деформируемого алюминиевого сплава марки Д16, наружным диаметром 100 мм и толщиной 2 мM.

Складкодержатель представлял собой плиту с габаритными размерами $100 \times 100 \times 5$ мм и центральным цилиндрическим отверстием диаметром 60 мм. Складкодержатель прижимался к фланцу листовой заготовки с силой 10 кН.

Пуансон изготавливался наружным диаметром 56 мм и общей длиной 120 мм. На торцевой кромке рабочей (давящей) части пуансона выполнялась радиусная фаска величиной 3 мм. На не рабочую торцевую поверхность пуансона действовало постоянное усилие величиной 40 кН.

Габаритные размеры вытяжной матрицы составляли $120 \times 120 \times 40$ мм. Сквозное цилиндрическое формообразующее отверстие вытяжной матрицы принималось величиной 70 мм. Таким образом, зазор между контактными поверхностями пуансона и рабочего отверстия вытяжной матрицы составил 7 мм. Размер радиусной фаски в рабочем отверстии вытяжной матрицы изменялся в диапазоне от 0.5 до 10.0 мм включительно с шагом 0.5 мм.

Складкодержатель, пуансон и вытяжная матрица по условию задачи принимались абсолютно твердыми телами.

Степень пластической деформации материала при различных величинах радиусной фаски в рабочем отверстии вытяжной матрицы представлена цветовыми контурами на моделях обрабатываемых заготовок (рис. 1).

Модель пуансона на эпюрах не изображалась. Цветовая шкала, справа от эпюр, определяет величину пластической деформации материала. 


\begin{tabular}{l|lrl|l|ll} 
& ISRA (India) & $=\mathbf{1 . 3 4 4}$ & SIS (USA) & $=\mathbf{0 . 9 1 2}$ & ICV (Poland) & $=\mathbf{6 . 6 3 0}$ \\
Impact Factor: & ISI (Dubai, UAE) $=\mathbf{0 . 8 2 9}$ & PUHU (Russia) $=\mathbf{0 . 1 7 9}$ & PIF (India) & $=\mathbf{1 . 9 4 0}$ \\
& GIF (Australia) & $\mathbf{0 . 5 6 4}$ & ESJI (KZ) & $=\mathbf{1 . 0 4 2}$ & & \\
& JIF & $=\mathbf{1 . 5 0 0}$ & SJIF (Morocco) $=\mathbf{2 . 0 3 1}$ & &
\end{tabular}

a)
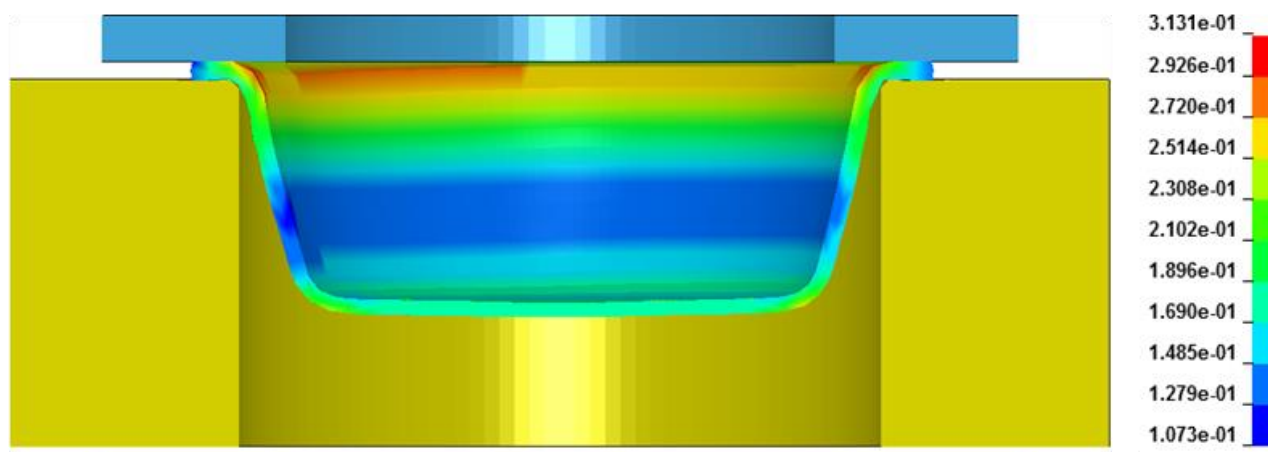

6)

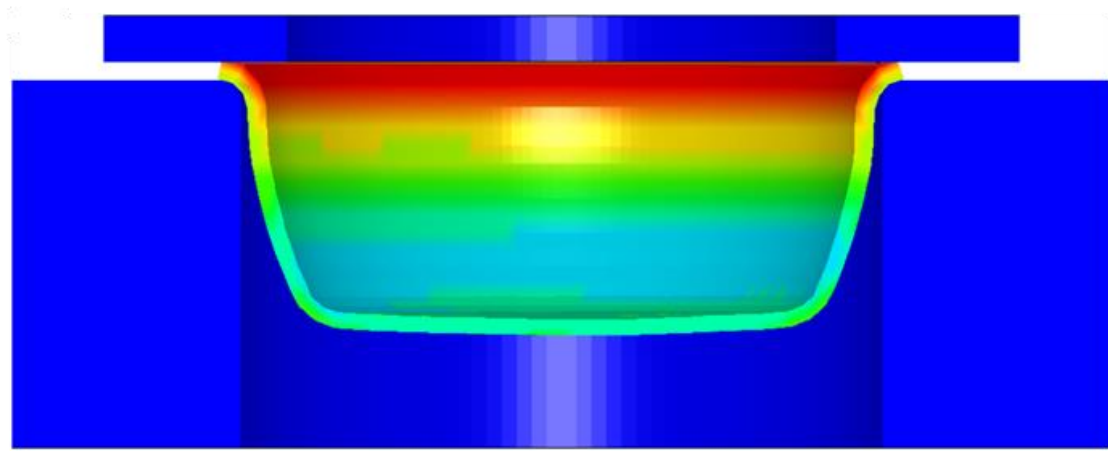

2.815e-01

2.534e- 01

2.252e- 01

1.971e-01

$1.689 \mathrm{e}-01$

$1.408 \mathrm{e}-01$

$1.126 \mathrm{e}-01$

8.446e-02

$5.630 \mathrm{e}-02$

$2.815 \mathrm{e}-02$

$0.000 e+00$

в)

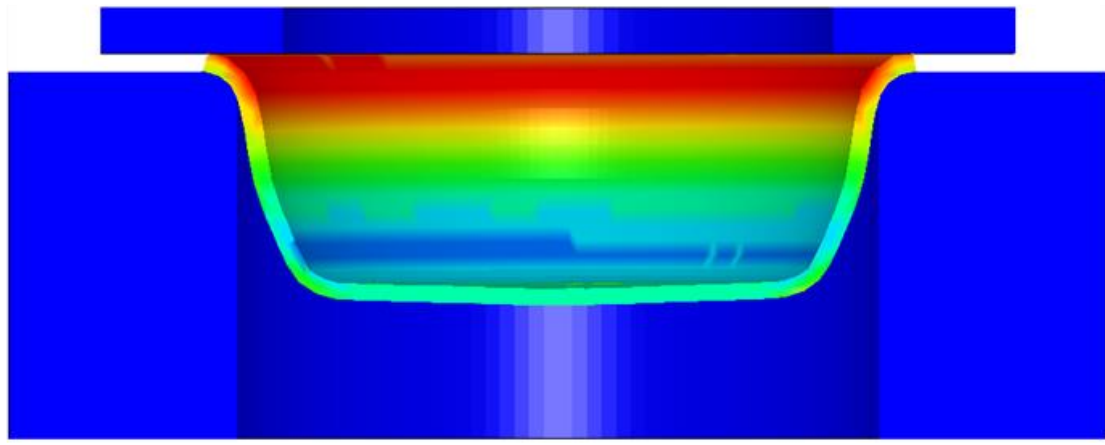

2.262e-01

2.035e-01

$1.809 \mathrm{e}-01$

$1.583 \mathrm{e}-01$

1.357e-01

1.131e-01

9.046e-02

$6.785 \mathrm{e}-02$

4.523e-02

$2.262 \mathrm{e}-02$

$0.000 e+00$

г)

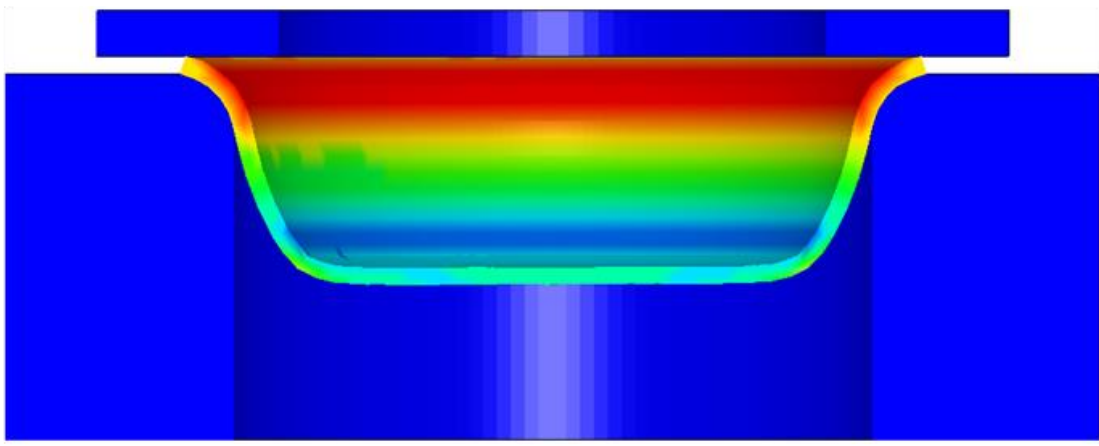

1.763e-01

$1.587 \mathrm{e}-01$

$1.411 \mathrm{e}-01$

$1.234 \mathrm{e}-01$

$1.058 \mathrm{e}-01$

$8.817 \mathrm{e}-02$

$7.054 \mathrm{e}-02$

$5.290 \mathrm{e}-02$

$3.527 \mathrm{e}-02$

$1.763 \mathrm{e}-02$

$0.000 \mathrm{e}+00$
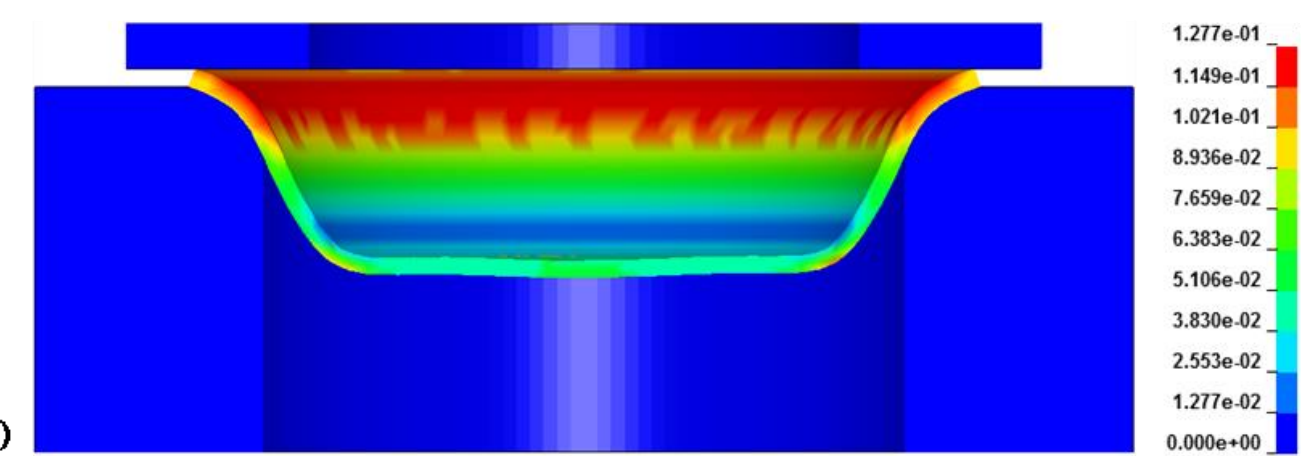

Рисунок 1 - Степень пластической деформации материала при изменении величины $r$ в отверстии

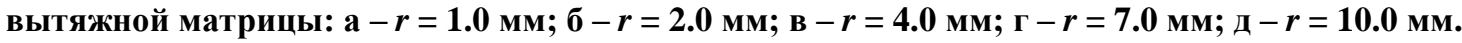

ISPC Perspectives in science for $\mathbf{2 0 1 6}$,

Philadelphia, USA

\section{THOMSON REUTERS}


Во всех случаях наибольшей пластической деформации подвергается верхняя часть вытягиваемой детали, наименьшей - нижняя часть.

При глубине вытяжки заготовки 25 мм (рис. 1 , а) толщина стенки в области радиусных фасок на рабочей части пуансона и в отверстии вытяжной матрицы утончается. Материал дальше не продавливается в отверстие вытяжной матрицы. Последующее движение пуансона вызовет разрыв металла в указанных местах. Максимальная величина пластической деформации составляет 0.313 .

С увеличением величины радиусной фаски в отверстии вытяжной матрицы (рис. 1, б - г) площадь деформации увеличивается, а значение коэффициента уменьшается до 0.176. Материал продавливается в рабочее отверстие вытяжной матрицы до формирования контура пустотелой детали.

При максимальном выбранном значении радиусной фаски (рис. 1 , д) происходит снижение пластической деформации материала до 0.127 (коэффициент меньше в 2.5 раза по сравнению с коэффициентом, полученным при $r=1.0$ мм). Однако в условии увеличения площади контакта материала с поверхностью формообразующего инструмента это приводит к образованию складок (гофр) [9].

Моделирование процесса глубокой вытяжки листовой заготовки с изменением величины радиусной фаски в отверстии матрицы показало:

1) при $r \leq 0.5 s$ вытяжка материала происходит не полностью со значительным утончением стенки, где $s$ - толщина листовой заготовки до обработки давлением, мм;

2) при $r=0.75 \mathrm{~s}$ вытяжка материала происходит полностью, однако контур детали получается несимметричным (не соответствует техническим требованиям на изготовление продукции);

3) при $r \geq s$ вытяжка материала происходит полностью без отклонений от технических требований на изготовление детали.

На рис. 2 представлен общий вид модели готовой пустотелой детали после глубокой вытяжки.

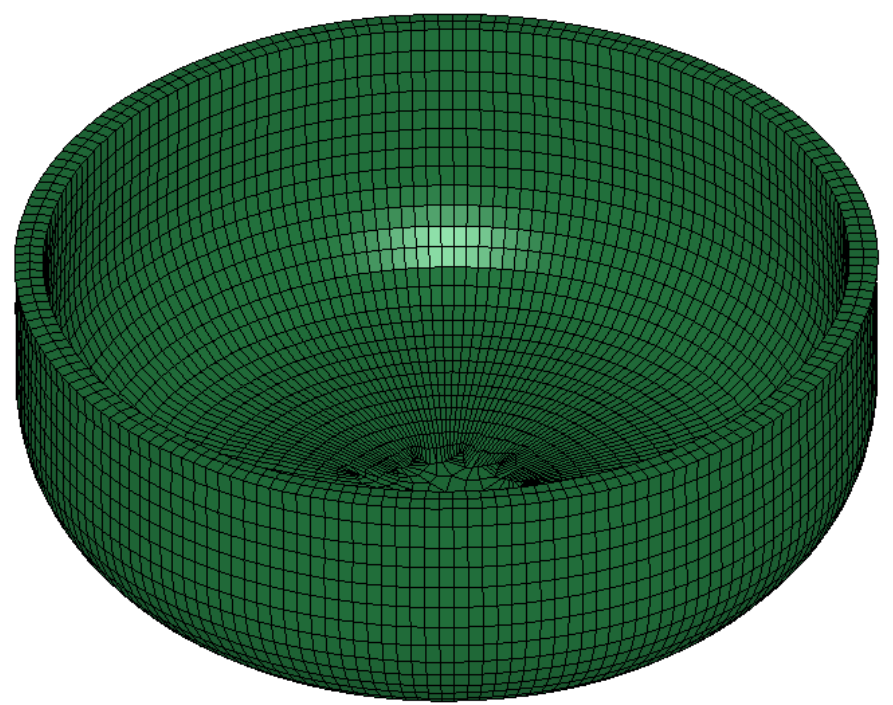

Рисунок 2 - Геометрическая форма полой детали после вытяжки.

Расчетная высота детали после вытяжки составляет 26 мм, радиус изгиба - 14 мм.

Значения времени процесса вытяжки детали $\tau$ в процентном выражении при различных величинах радиусной фаски в отверстии вытяжной матрицы представлены в табл. 1.
Прочерки в таблице означают, что значения времени обработки детали для данного диапазона величин радиусной фаски не определялись (производственные дефекты). За 100\% принято лимитирующее время обработки детали.

\section{Время вытяжки детали в процентном выражении.}

\section{Таблица 1}

\begin{tabular}{|c|c|c|c|c|c|c|c|c|c|c|}
\hline$r, \mathrm{MM}$ & 0.5 & 1.0 & 1.5 & 2.0 & 2.5 & 3.0 & 3.5 & 4.0 & 4.5 & 5.0 \\
\hline$\tau, \%$ & - & - & - & 100 & 98.3 & 93.6 & 93.6 & 88.7 & 90.3 & 90.3 \\
\hline$r, \mathrm{MM}$ & 5.5 & 6.0 & 6.5 & 7.0 & 7.5 & 8.0 & 8.5 & 9.0 & 9.5 & 10.0 \\
\hline$\tau, \%$ & 89.5 & 88.7 & 88.7 & 88.7 & 87.1 & 87.1 & 87.1 & 87.1 & 88.7 & 90.3 \\
\hline
\end{tabular}

ISPC Perspectives in science for $\mathbf{2 0 1 6}$ 


\begin{tabular}{|c|c|c|c|c|c|c|}
\hline Impact Factor: & $\begin{array}{l}\text { ISRA (India) } \\
\text { ISI (Dubai, UAE } \\
\text { GIF (Australia) } \\
\text { JIF }\end{array}$ & $\begin{array}{l}=1.344 \\
=0.829 \\
=0.564 \\
=1.500\end{array}$ & $\begin{array}{l}\text { SIS (USA) } \\
\text { PИНЦ (Russia) } \\
\text { ESJI (KZ) } \\
\text { SJIF (Morocco) }\end{array}$ & $\begin{array}{l}=0.912 \\
=0.179 \\
=1.042 \\
=2.031\end{array}$ & $\begin{array}{l}\text { ICV (Poland) } \\
\text { PIF (India) }\end{array}$ & $\begin{array}{l}=6.630 \\
=1.940\end{array}$ \\
\hline
\end{tabular}

Зависимости работы деформирования заготовки при вытяжке от времени процесса, экономии времени обработки детали и изменения значения коэффициента пластической

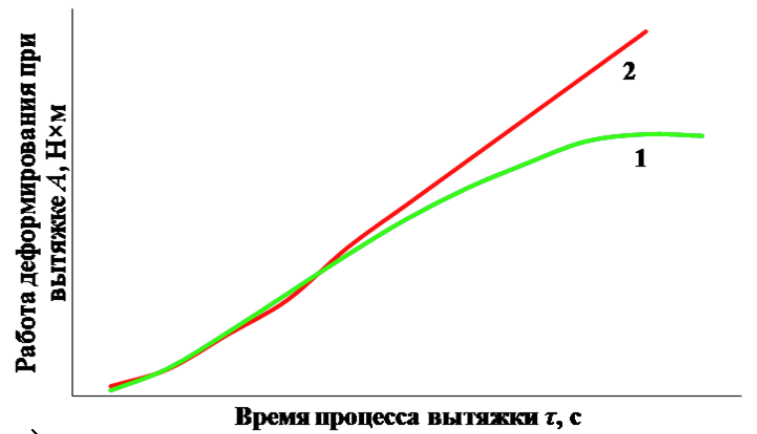

a)

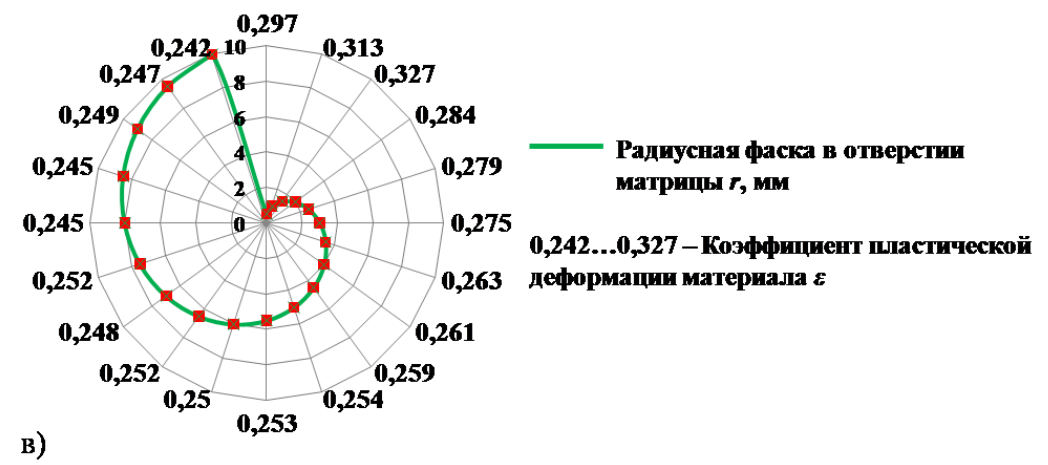

Рисунок 3 - Анализ результатов расчета, представленный в графическом виде: а - зависимости работы деформирования заготовки при вытяжке (на графике 1 - для r0.5 мм, 2 - для $r 10$ мм) от времени процесса; б - зависимости экономии времени обработки детали от величины радиусной фаски в отверстии матрицы; в - изменение значения коэффициента пластической деформации материала от величины радиусной фаски в отверстии матрицы.

На основании проведенного исследования процесса глубокой вытяжки можно сделать следующие выводы:

1. Затраты энергии на работу деформирования листовой заготовки зависит от величины радиуса фаски - чем больше радиус, тем больше потребляется мощность технологического оборудования.

2. Определен оптимальный диапазон величин радиусных фасок, позволяющий экономить время обработки детали. В случае заготовки толщиной 2 мм этот диапазон деформации материала от величины радиусной фаски в отверстии матрицы представлены на рис. 3 ,

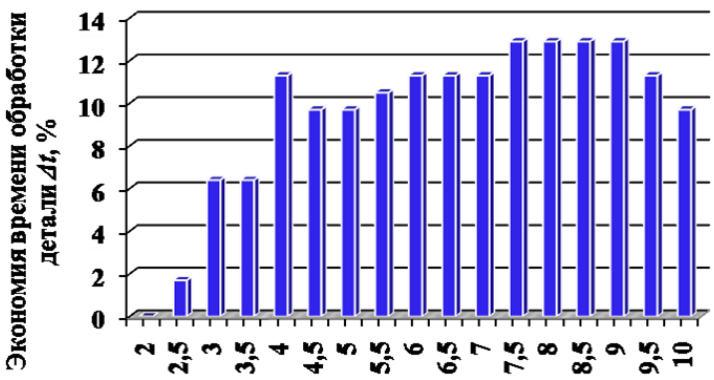

б) 


\begin{tabular}{l|lrl|l|ll} 
& ISRA (India) & $=\mathbf{1 . 3 4 4}$ & SIS (USA) & $=\mathbf{0 . 9 1 2}$ & ICV (Poland) & $=\mathbf{6 . 6 3 0}$ \\
Impact Factor: & ISI (Dubai, UAE) $=\mathbf{0 . 8 2 9}$ & PUHL (Russia) $=\mathbf{0 . 1 7 9}$ & PIF (India) & $=\mathbf{1 . 9 4 0}$ \\
& GIF (Australia) & $\mathbf{0 . 5 6 4}$ & ESJI (KZ) & $=1.042$ & & \\
& JIF & $\mathbf{1 . 5 0 0}$ & SJIF (Morocco) $=\mathbf{2 . 0 3 1}$ & &
\end{tabular}

2. Chemezov DA, Seliverstov VS (2015) The intensity of the formation of corrugation on the flange of the deformable plate stock of thickness $1-5 \mathrm{~mm}$. Scientific and theoretical journal «Systems Engineering», №2. - pp. 71 76. ISSN 2304-0823.

3. Chemezov DA (2015) The research of the shallow drawing process of the plate stock. ISJ Theoretical \& Applied Science 10 (30): 11-15. Soi: http://s-o-i.org/1.1/TAS-10-30-4 Doi: http://dx.doi.org/10.15863/TAS.2015.10.30.4

4. Chemezov DA, Zezina NA, Seliverstov VS (2015) The determination of the bending moment at the pressure of the punch on the material in the conditions of the shallow drawing of the plate stock. XII International scientific-practical conference "Fundamental and applied researches in the modern world". Vol. 1. - pp. 73 - 76. ISSN 2307-1354.

5. Chemezov DA, Seliverstov VS, Komisar AS, Zezina NA, Tyurina SI (2015) Stamping of the plate stock with blank holder: the character of the material deformation and calculation of the coefficient of elongation. ISJ Theoretical \& Applied Science 11 (31): 101-107. Soi: http://so-i.org/1.1/TAS-11-31-16

Doi: http://dx.doi.org/10.15863/TAS.2015.11.31.16

6. Chemezov DA (2015) Changing the wall thickness of the hollow detail during a shallow drawing of the plate stock. ISJ Theoretical \& Applied Science 12 (32): 34- 37. Soi: http://s-oi.org/1.1/TAS-12-32-5 Doi: http://dx.doi.org/10.15863/TAS.2015.12.32.5

7. (2016) Klassiphikatsiya ostatochnyh napryazheny $\mathrm{v}$ metalle i metody ih izmereniya. Available: (Accessed: 13.01.2016).

8. (2016) Teoriya OMD. Available: http://anybook.org/download/18120.html (Accessed: 13.01.2016).

9. (2016) Radiusy zakrugleny i zazory pri vytyazhke. Available: http://omd.omgtu.ru/wpcontent/themes/iGadgets/basetlsh/178-184.htm (Accessed: 13.01.2016). 